Check for updates

Cite this: Mater. Adv., 2020, 1, 3565

Received 9th September 2020, Accepted 13th November 2020

DOI: $10.1039 / \mathrm{d} 0 \mathrm{ma00691b}$

rsc.li/materials-advances

\title{
Self-assembly pattern directed sustained release from porous microspheres of discotic tripeptides $\dagger$
}

\begin{abstract}
Santosh Kumar, Santu Bera, (D) Sujay Kumar Nandi and Debasish Haldar (D) *
Porous microspheres from discotic tripeptides have been investigated as potential candidates for drug delivery vehicles. The $C_{3}$ symmetric discotic tripeptide adopts a supramolecular helical column structure by three fold intermolecular hydrogen bonding interactions as well as face to face $\pi-\pi$ stacking interactions. But the $C_{2}$ symmetric discotic tripeptide adopts a supramolecular dimer like structure by six-fold intermolecular hydrogen bonding interactions and face to face $\pi-\pi$ stacking interactions. Field emission scanning electron microscopy (FE-SEM) revealed that the $C_{3}$ symmetric discotic tripeptide exhibits bird nest-like porous microsphere morphology formed by the assembly of the individual columns. However the $C_{2}$ symmetric discotic tripeptide forms round clay pitcher like porous microspheres. These porous microspheres have been used as potential carriers for the sustained release of a bacteriostatic antibiotic sulfamethoxazole. The spectroscopic studies as well as the growth inhibition of $E$. coli reveal that the round clay pitcher-like porous microspheres are more efficient than the bird nest-like porous microspheres for the sustained release of drugs. The report highlights the importance of the selfassembly pattern for the fabrication of advanced functional materials.
\end{abstract}

\section{Introduction}

Supramolecular microstructures developed via self-assembly of building blocks have received considerable attention because these structures can lead to advanced functional materials. ${ }^{1-5}$ The self-assembly process is governed by various non covalent interactions, including hydrogen-bonding interactions, ion-pair interactions, and $\pi-\pi$ stacking and hydrophobic interactions. ${ }^{6-8}$ Normally, this self-assembly process is fully reversible which leads to numerous possible applications of the supramolecular material in the fields of environmental sciences, material sciences, biomedical sciences and chemistry. ${ }^{9-12}$ The reversibility of the supramolecular systems has led to a change in their topologies and properties upon exposure to external stimuli such as temperature, light, $\mathrm{pH}$, and redox potential. ${ }^{13-15}$ Among these diverse self-assembly systems, the amphiphilic microspheres offer potential applications for drug encapsulation, delivery, and sustained release. ${ }^{16}$

Unwanted side effects and systemic toxicity are common hazards of using naked drugs. ${ }^{17}$ So, rapping and pharmaceutical formulation is important. ${ }^{18}$ But this should work in synergy and

Department of Chemical Sciences, Indian Institute of Science Education and Research Kolkata, Mohanpur, West Bengal 741246, India.

E-mail: deba_h76@yahoo.com,deba_h76@iiserkol.ac.in; Fax: +91 3325873020;

Tel: +913325873119

$\dagger$ Electronic supplementary information (ESI) available: Synthesis and characterization of nitropeptides, ${ }^{1} \mathrm{H}$ NMR, ${ }^{13} \mathrm{C}$ NMR, Fig. S1-S4 and S5-S10 or other electronic format. See DOI: 10.1039/d0ma00691b will not generate toxic chemicals by further reaction. An ideal delivery vehicle should deliver the required amount of drug to the target site and confirm the sustained release of the encapsulated drug under physiological conditions. ${ }^{19}$ To date, diverse compounds such as polymers and polymer-based micelles, ${ }^{20}$ metal-organic frameworks (MOFs), ${ }^{21}$ covalent organic frameworks (COFs), ${ }^{22}$ carbon nanotubes, ${ }^{23}$ metal nanoparticles, ${ }^{24}$ silica, ${ }^{25}$ iron oxide nanoparticles, ${ }^{26}$ or dendrimer nanoparticles have been used for drug formulation and delivery. ${ }^{27}$ But, due to surface modification most of these reported delivery systems have side effects including intrinsic toxicity or immunogenicity. ${ }^{28}$ In this regard, peptide-based self-assembled microspheres ${ }^{29}$ are promising due to their biocompatibility ${ }^{30}$ and recognition properties. ${ }^{31}$ Also, due to their hydrophilic-hydrophobic balance for solubilising and targeted delivery of highly hydrophobic anti-tumour ${ }^{32}$ and antidepressant drugs, ${ }^{33}$ and statins, ${ }^{34}$ peptide-based delivery vehicles are very popular. Moreover, the hybrid peptides have stability towards enzymatic degradation. ${ }^{35}$ Hence, the design and synthesis of new peptide building blocks and delivery vehicles are of great interest. P. K. Das and co-workers have reported vesicles from discotic amphiphiles that can encapsulate and deliver doxorubicin inside mammalian cells. ${ }^{36}$

We are developing microspheres and microvesicles as delivery vehicles, from peptidomimetic compounds. ${ }^{37}$ Herein, we have designed and synthesized two discotic tripeptides with different symmetries, from commercially available sources. The discotic tripeptide having $C_{3}$ symmetry adopts a supramolecular 
column structure stabilized by three-fold intermolecular hydrogen bonds. However, the discotic tripeptide having $C_{2}$ symmetry forms a supramolecular dimer stabilized by six-fold intermolecular hydrogen bonding interactions. The FE-SEM and the Atomic Force Microscopy (AFM) studies revealed that the $C_{3}$ symmetric discotic tripeptide shows bird nest like porous microsphere morphology but the $C_{2}$ symmetric discotic tripeptide exhibits round clay pitcher like porous microsphere morphology. The microspheres were loaded with the bacteriostatic antibiotic sulfamethoxazole. The round clay pitcher-like porous microspheres of the $C_{2}$ symmetric discotic tripeptide are more efficient than the bird nest-like porous microspheres of the $C_{3}$ symmetric discotic tripeptide, for the sustained release of encapsulated drugs.

\section{Results and discussion}

\section{Synthesis and characterization}

We have designed and synthesized two discotic tripeptides having $C_{3}$ symmetry and $C_{2}$ symmetry (Scheme 1 ). The assumption was that compound 1 with $C_{3}$ symmetry would form a supramolecular columnar structure by three-fold intermolecular hydrogen bonds between amide functionalities, like that reported in earlier reports. ${ }^{36}$ However, compound 2 with $C_{2}$ symmetry may fail to form such a columnar structure. Three L-Phe residues should impart hydrophobicity in the compounds and direct donor-acceptor type $\pi-\pi$ stacking and further modulate the self-assembly process. The discotic tripeptide 1 was synthesized from benzene-1,3,5-tricarboxylic acid and L-phenylalanine methyl ester using DCC as a coupling reagent in $72 \%$ yields. The discotic tripeptide 2 was synthesized by coupling methyl ester of 5-aminoisophthalic acid and Boc-Phe-OH followed by hydrolysis and further coupling with L-phenylalanine methyl ester using DCC as a coupling reagent in $44.5 \%$ yield (Scheme S1, ESI $\dagger$ ). All the synthesized discotic tripeptides and intermediates were purified by column chromatography and<smiles>[R1]NC(=O)c1cc(C(=O)N[R1])cc(C(=O)N[R1])c1</smiles>

1

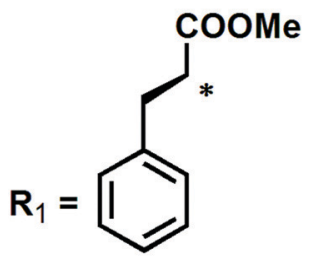

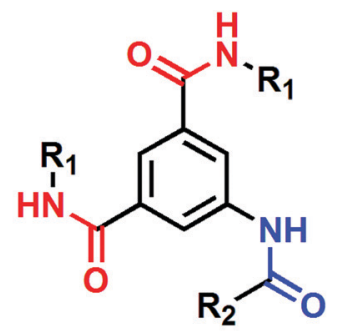

2

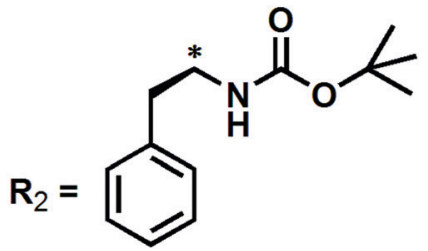

Scheme 1 The schematic presentation of discotic tripeptides 1 and 2 . fully characterized by ${ }^{1} \mathrm{H}-\mathrm{NMR},{ }^{13} \mathrm{C}-\mathrm{NMR}$ and FTIR spectroscopy and mass spectrometry.

\section{Morphology}

The self-assembly propensity of the discotic tripeptides $\mathbf{1}$ and $\mathbf{2}$ was examined by field emission scanning electron microscopy (FE-SEM) and atomic force microscopy (AFM). For FE-SEM studies, a small amount of solution $(0.02 \mathrm{M})$ of the discotic tripeptides 1 and 2 in methanol was placed on a clean glass slide and then dried by slow evaporation at room temperature. The samples were then allowed to dry under vacuum at $30{ }^{\circ} \mathrm{C}$ for $24 \mathrm{~h}$. The dried samples were coated with gold, and the micrographs were taken in a Zeiss DSM 950 scanning electron microscope. The FE-SEM images of the discotic tripeptide 1 exhibit the formation of bird nest-like polydisperse porous microsphere morphology (Fig. 1a). The average diameter of the microspheres is about $1.5 \mu \mathrm{m}$. A careful observation revealed that the bird nest-like porous microspheres are formed by assembly of nanorods (Fig. 1b). The average diameter of the nanorods is $c a .130 \mathrm{~nm}$. The size of the pores on the surface of the microspheres is $c a .50 \mathrm{~nm}$. We were also able to capture the image of bird nest-like porous microspheres formed from the nanorods (Fig. S1, ESI $\dagger$ ), which shows that the shape and size of the nanorods are very regular. However, the FE-SEM images of the discotic tripeptide 2 show the formation of round clay pitcher like polydisperse porous microsphere morphology (Fig. 1c). The average diameter of the microspheres is ca. $1.2 \mu \mathrm{m}$. The internal diameter of the hollow inside the microspheres is $c a .800 \mathrm{~nm}$. The thickness of the microsphere wall is ca. $150 \mathrm{~nm}$. The inset of Fig. 1d showing an enlarged image of a microsphere like round clay pitcher clearly indicates the presence of cavities inside the microspheres and pores on the surface. The size of the pore is $c a .50 \mathrm{~nm}$. To know about the

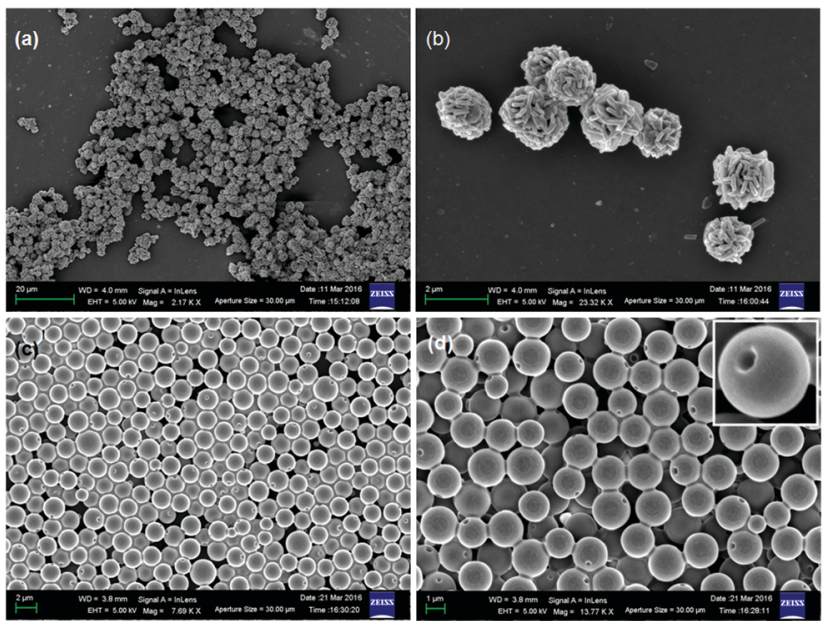

Fig. 1 (a) The FE-SEM images showing bird nest-like porous microsphere morphology of discotic tripeptide 1. (b) The FE-SEM images showing the assembly of nanorods to form the bird nest-like porous microspheres of discotic tripeptide 1. (c) and (d) The FE-SEM images showing round clay pitcher like porous microsphere morphology of discotic tripeptide 2 . (d) The inset showing the pores of the microspheres of discotic peptide $\mathbf{2}$. 
topography of the discotic tripeptides 1 and 2, atomic force microscopic studies have been performed. For AFM studies, the solutions $(0.02 \mathrm{M})$ of tripeptides $\mathbf{1}$ and 2 in methanol were placed on a microscopic glass slide and allowed to dry under vacuum at $30{ }^{\circ} \mathrm{C}$ for two days and studied using a NT-MDT atomic force microscope. The AFM micrograph of discotic tripeptide 1 shows the polydisperse microsphere like morphology where the surface of the microspheres is very rough (Fig. S2a, ESI $\dagger$ ). Fig. S2b (ESI $\dagger$ ) shows the 3D AFM image of the microsphere morphology. However, the AFM image of discotic tripeptide 2 shows the polydisperse microsphere like morphology where the surface of the microspheres is quite smooth (Fig. S2c, ESI $\dagger$ ). Fig. S2d (ESI $\dagger$ ) shows the 3D AFM image of the microsphere morphology with a smooth surface.

\section{Structure analysis}

For discotic tripeptide 2, mass spectrometry points to the existence of both the monomer and dimer. Distinct signals for the monomer and dimer were detected using a Q-Tof Micro YA263 high-resolution mass spectrometer (Fig. 2).

Circular dichorism (CD) spectroscopy in the solution state was performed to study the aggregation propensity of the discotic tripeptides 1 and 2 . The shape and intensity of the CD spectra differ significantly for discotic tripeptides $\mathbf{1}$ and $\mathbf{2}$ in methanol. Discotic tripeptide 1 shows a positive cotton effect at $198 \mathrm{~nm}$ and negative cotton effects at $208 \mathrm{~nm}$ and $228 \mathrm{~nm}$ (Fig. 3a), reflecting the formation of helical supramolecular stacks. ${ }^{38}$ We conclude that such a strong CD signal originates from a rigid, elongated columnar self-assembly of the compound. The discotic tripeptide 2 has a positive band at $200 \mathrm{~nm}$ and negative bands at 211 and $226 \mathrm{~nm}$ with much lower intensity responsible for a dimer structure of shorter stacking (Fig. 3a).

Solid-state FT-IR spectroscopy was performed to study the structure of the discotic tripeptides $\mathbf{1}$ and $\mathbf{2}$ in porous microspheres. For discotic tripeptide 1, an intense band at $1743 \mathrm{~cm}^{-1}$ indicates the presence of three ester groups in the compound (Fig. 3b). ${ }^{39}$ The peaks at $1641 \mathrm{~cm}^{-1}$ and $1537 \mathrm{~cm}^{-1}$ arise due to the formation of $\mathrm{H}$-bonded stacks of the amide $\mathrm{C}=\mathrm{O}$ groups (Fig. 3b). For discotic tripeptide 2, the band at $1739 \mathrm{~cm}^{-1}$ indicates the presence of two ester groups (Fig. 3b). The peaks at $1660 \mathrm{~cm}^{-1}$ and $1528 \mathrm{~cm}^{-1}$ arise due to the formation of $\mathrm{H}$-bonded amide I and amide II (Fig. $3 \mathrm{~b}$ ). Another informative

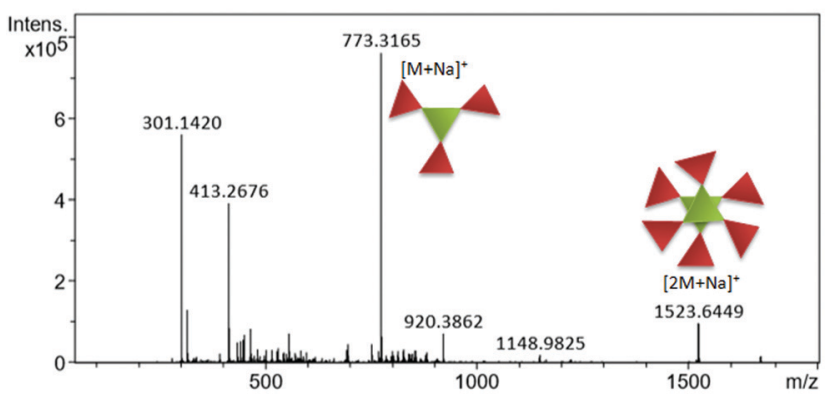

Fig. 2 Mass spectrometry data showing the coexistence of both the monomer and dimer of discotic tripeptide $\mathbf{2}$.
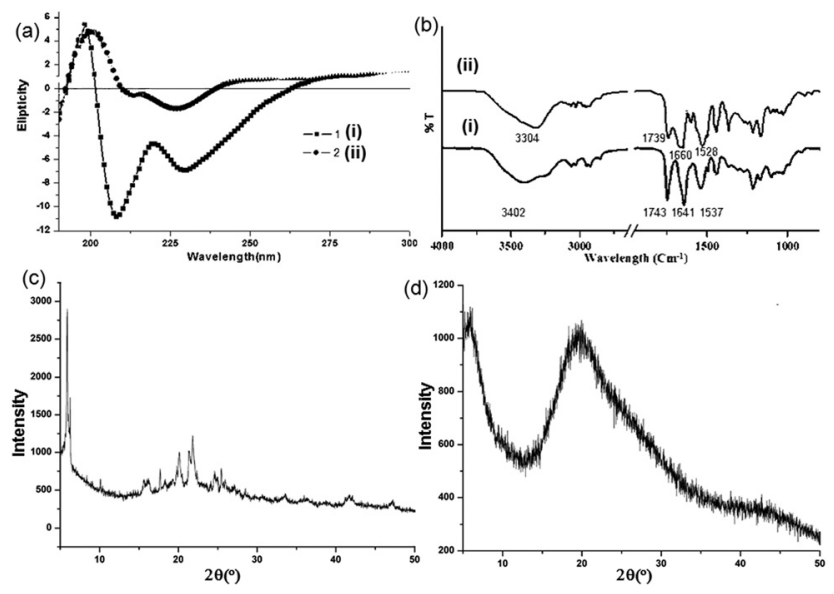

Fig. 3 (a) CD spectra of the discotic tripeptides 1 and $\mathbf{2}$ in methanol. (b) Solid-state FT-IR spectra of the microspheres obtained from discotic tripeptides 1 and 2. (c) and (d) PXRD patterns of discotic tripeptides 1 and 2 , respectively.

frequency range is $3500-3200 \mathrm{~cm}^{-1}$, corresponding to the $\mathrm{N}-\mathrm{H}$ stretching vibration. The discotic tripeptides 1 and 2 show peaks at $3304 \mathrm{~cm}^{-1}$ for hydrogen-bonded NH (Fig. 3b).

The structures of the discotic tripeptides 1 and 2 were also studied by powder X-ray diffraction (PXRD). The PXRD pattern of the microspheres of discotic tripeptide 1 shows that the material is crystalline in nature. Sharp reflections were observed in the $5-50^{\circ} 2 \theta$ range (Fig. 3c). Powder X-ray diffraction (PXRD) had reflection at $d$-spacings of $4.07 \AA$ and $15.98 \AA$ corresponding to the distances between neighbouring aromatic moieties and the laminal spacing, respectively. However, the PXRD pattern of the microspheres of discotic tripeptide 2 shows that the material is non-crystalline in nature (Fig. 3d).

From the above experimental results, we propose that the discotic tripeptide 1 self-assembles by three-fold intermolecular hydrogen bonding interactions to form supramolecular helical columnar stacks which further bundled to develop a nano rodlike structure (Fig. 4a). In higher-order, these nanorods are assembled to form bird nest-like porous microspheres (Fig. 4a). However, the discotic tripeptide 2 self-assembles by six-fold intermolecular hydrogen bonding interactions to form a dimer which further assembles to develop a 2D sheet-like structure that folds and forms the round clay pitcher like porous microspheres (Fig. 4b).

The microspheres from discotic tripeptides are stable and insoluble in water. We have encapsulated a bacteriostatic antibiotic sulfamethoxazole in discotic tripeptide $\mathbf{1}$ and $\mathbf{2}$ microspheres. To $3 \mathrm{~mL}$ of $0.50 \times 10^{-3} \mathrm{M}$ drug solution in methanol, $5 \mathrm{mg}$ of discotic tripeptides 1 or 2 was added and stirred for $6 \mathrm{~h}$, the solvent was evaporated under vacuum and the drug-loaded microspheres were washed several times with $50 \mathrm{~mL}$ water and centrifuged until the drained water after centrifugation exhibited negligible drug fluorescence. The drug-binding property of the microspheres obtained from methanol solution of discotic tripeptides 1 or 2 was studied by absorption and emission spectroscopy as these are quite sensitive techniques to understand any changes in 


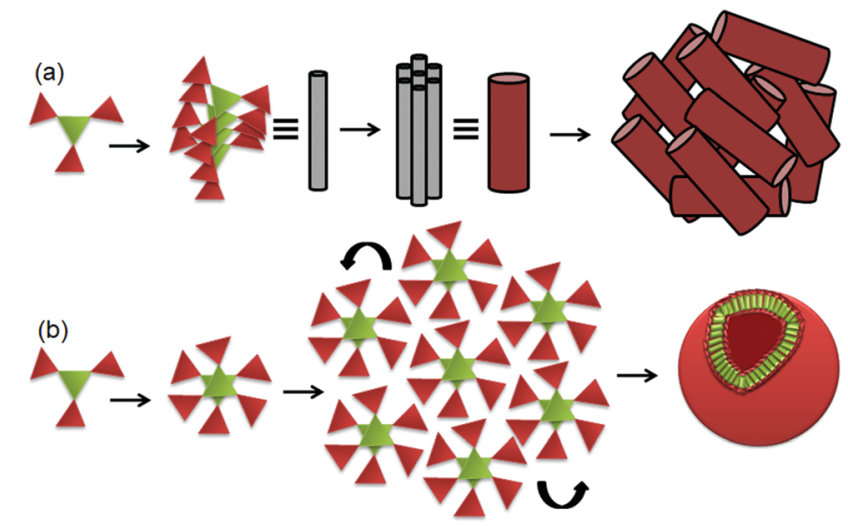

Fig. 4 (a) The schematic presentation, showing self-assembly of discotic tripeptide 1 to columnar stacks to nanorods to porous microspheres. (b) The schematic presentation showing self-assembly of discotic tripeptide 2 to dimers to $2 \mathrm{D}$ sheets to porous microspheres.

microenvironments. From the absorption spectrum of discotic tripeptide 2 (Fig. 5a), it is clear that with increasing sulfamethoxazole concentration the absorbance at $262 \mathrm{~nm}$ gradually increases and the spectrum is red shifted $(c a .6 \mathrm{~nm})$. This implies that the microspheres have some interactions with the drug molecules. The result was further supported by emission spectroscopy. From Fig. 5b, it is evident that when we increase the concentration of the drug the fluorescence intensity of discotic tripeptide 2 at $347 \mathrm{~nm}$ gradually decreases and this spectrum is also shifted by $10 \mathrm{~nm}$. Discotic tripeptide $\mathbf{1}$ also shows similar results with increasing sulfamethoxazole concentration studied by absorption spectroscopy (Fig. S3, ESI $\dagger$ ).

The encapsulation efficiency (\%) was quantified by absorption spectroscopy and calculated as [(amount of drug added amount of free drug)/amount of drug added] $\times 100 \%$. The encapsulation efficiency of the drug loaded discotic tripeptide 1 microspheres was found to be $60 \%$ and that of discotic tripeptide 2 microspheres was $70 \%$. The drug loading content for the formulation was calculated as [weight of the encapsulated drug in the microspheres/weight of the microspheres used] $\times 100 \%$ and was found to be $4.33 \%$ for discotic tripeptide 1 and $5.33 \%$ for discotic tripeptide 2 .

A further FE-SEM experiment was performed to know whether there is any change in the size or shape of the microspheres after
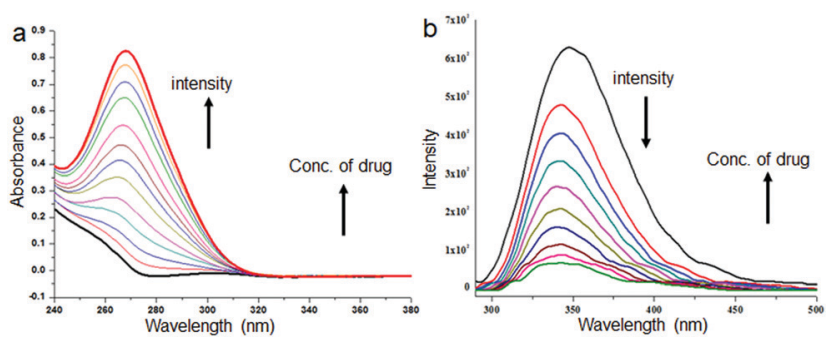

Fig. 5 (a) Absorption spectra of discotic tripeptide 2 with increasing concentration of sulfamethoxazole in methanol. Discotic tripeptide 2 concentration $=1 \times 10^{-5} \mathrm{M}$. (b) Emission spectra of discotic tripeptide 2 with increasing concentration of sulfamethoxazole in methanol. Excitation wavelength is $270 \mathrm{~nm}$. Discotic tripeptide 2 concentration $=1 \times 10^{-5} \mathrm{M}$.

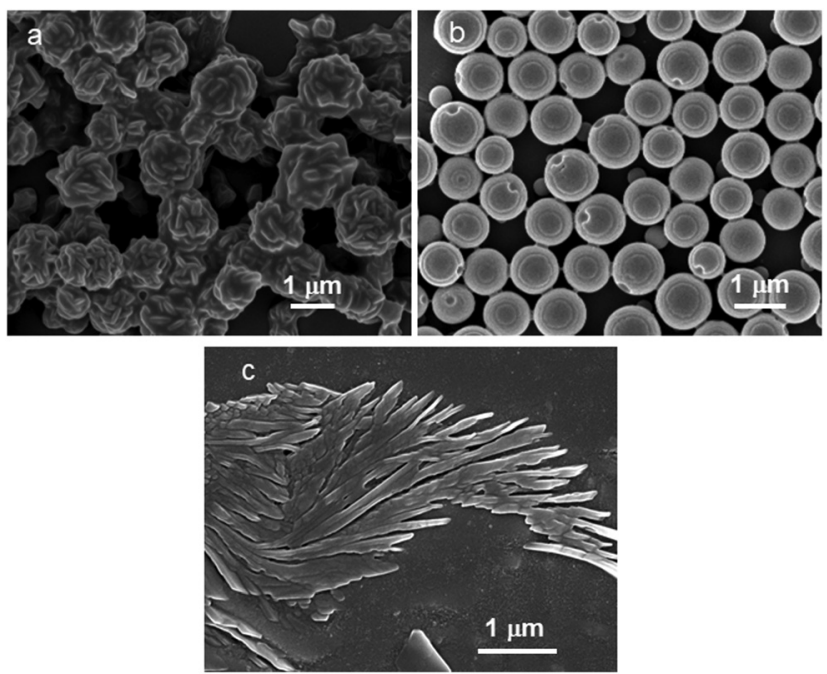

Fig. 6 (a) FE-SEM image of discotic tripeptide 1 polydisperse bird nest like microspheres after sulfamethoxazole loading. (b) FE-SEM image of discotic tripeptide $\mathbf{2}$ polydisperse microspheres after sulfamethoxazole encapsulation. (c) FE-SEM image exhibiting fiber like morphology of sulfamethoxazole.

drug loading. The FE-SEM image of the drug-loaded discotic tripeptide 1 clearly indicates that the bird nest-like microsphere morphology is retained and there is no average change in size and shape after drug encapsulation (Fig. 6a). Only the pores are absent in the drug-loaded microspheres. Fig. 6b shows the FE-SEM image of the drug encapsulated discotic tripeptide 2 microspheres, which clearly indicates that the morphology is retained, only the pores of the microspheres are filled up. For reference, an FE-SEM study of only the sulfamethoxazole drug under the same conditions was done. Fig. 6c shows the fiber-like morphology of sulfamethoxazole. The fibers have a diameter of $c a .100 \mathrm{~nm}$ and are several micrometers in length.

We have also studied the release of the encapsulated sulfamethoxazole from the nanovesicles. For this purpose, sulfamethoxazole loaded nanovesicles were immersed into $5 \mathrm{~mL}$ Tris buffer (at pH 7.4) in a $15 \mathrm{~mL}$ centrifuge tube, centrifuged at $2800 \mathrm{rpm}$ for 15 minutes, and examined by absorption spectroscopy at different time intervals. Fig. 7a shows the drug release profile which clearly indicates that the microspheres of the discotic tripeptide 2 slowly release the encapsulated drug compared to the microspheres of the discotic tripeptide 1 , with a complete release by $30 \mathrm{~h}$ (Fig. S4, ESI $\dagger$ ).

The sustainable release of encapsulated sulfamethoxazole and in vitro antibacterial activity in water and DMSO (10\%) on E. coli was examined by OD measurement at $600 \mathrm{~nm} \cdot{ }^{40}$ Fig. $7 \mathrm{~b}$ shows the growth inhibition profile of $E$. coli against the sulfamethoxazole solution in $10 \%$ DMSO-water. E. coli is more susceptible in sulfamethoxazole encapsulated microsphere solution than the naked sulfamethoxazole solution (control) (Fig. 7b). It is clear from the diagram that $E$. coli is more susceptible to the drug-loaded microspheres of discotic tripeptide 2 than the drug-loaded microspheres of discotic tripeptide 1 (Fig. 7b). Fig. 7c(i) and (ii) show the growth inhibition zones of $E$. coli against the drugloaded microspheres of discotic tripeptides 1 and 2, respectively, 

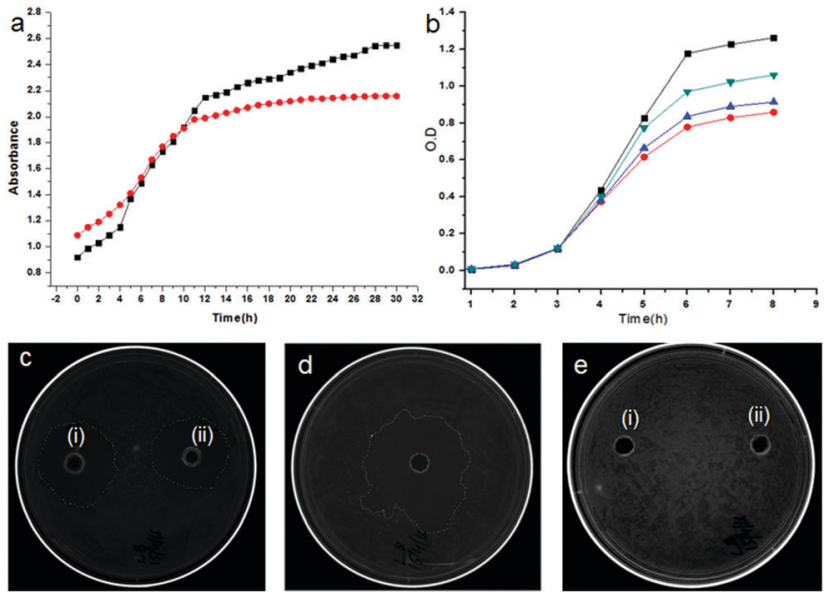

Fig. 7 (a) Drug release profile of sulfamethoxazole loaded microspheres of discotic tripeptide 1 (red) and sulfamethoxazole loaded microspheres of discotic tripeptide 2 (black) in tris buffer $(\mathrm{pH}$ 7.4) obtained from UV-Vis spectroscopy. (b) The growth inhibition plot of E. coli with only drug (red), discotic tripeptide 1-drug (blue), discotic tripeptide 2-drug (dark cyan), and without any drug (black). The growth inhibition zones of E. coli bacteria against the (c) (i) encapsulated discotic tripeptide 1-drug, (c) (ii) encapsulated discotic tripeptide 2-drug, (d) sulfamethoxazole (drug), (e) (i) discotic tripeptide 1 and (e) (ii) discotic tripeptide 2 in 10\% DMSO-water.

and Fig. $7 d$ shows the same for naked sulfamethoxazole in $10 \%$ DMSO-water. Furthermore, Fig. 7e indicates that the discotic tripeptides $\mathbf{1}$ and $\mathbf{2}$ do not have any antibacterial activity on the E. coli bacteria.

\section{Conclusions}

In conclusion, this study demonstrates the synthesis and selfassembly of $C_{2}$ and $C_{3}$ symmetric discotic tripeptides. The $C_{3}$ symmetric discotic tripeptide forms a supramolecular columnar structure by three fold intermolecular hydrogen bonds. But the $C_{2}$ symmetric discotic tripeptide adopts a supramolecular dimer by six fold intermolecular hydrogen bonds. FE-SEM images show that the $C_{3}$ symmetric discotic tripeptide exhibits bird nest-like porous microsphere morphology formed by the assembly of the columns. However the $C_{2}$ symmetric discotic tripeptide forms round clay pitcher like porous microspheres. Furthermore, these microspheres were loaded with the antibiotic sulfamethoxazole. However, the discotic tripeptides are not growth inhibitors of $E$. coli. The spectroscopic studies as well as the growth inhibition of $E$. coli reveal that the round clay pitcher-like porous microspheres are more efficient than the bird nest-like porous microspheres for the sustained release of drugs. The results presented here show the importance of the self-assembly pattern for the fabrication of delivery vehicles.

\section{Experimental}

General

All chemicals were purchased from Sigma chemicals. The discotic peptides were synthesized by conventional solution phase methodology (ESI $\dagger$ ). All the products were purified by column chromatography using silica (100-200 mesh size) gel as a stationary phase and an $n$-hexane-ethyl acetate mixture as an eluent. The intermediates and final compounds were fully characterized by $400 \mathrm{MHz}{ }^{1} \mathrm{H}$ NMR spectroscopy, $100 \mathrm{MHz}$ ${ }^{13} \mathrm{C}$ NMR spectroscopy and FTIR spectroscopy and mass spectrometry.

\section{NMR experiments}

All NMR studies were carried out on a JEOL $400 \mathrm{MHz}$ spectrometer at $25{ }^{\circ} \mathrm{C}$. Compound concentrations were in the range of 1-10 $\mathrm{mM}$ in $\mathrm{CDCl}_{3}$ and $\left(\mathrm{CD}_{3}\right)_{2} \mathrm{SO}$.

\section{FTIR spectroscopy}

The solid-state FTIR spectrum was obtained with a PerkinElmer Spectrum RX1 spectrophotometer with the KBr disk technique.

\section{UV/Vis spectroscopy}

UV/Vis absorption spectra were recorded on a UV/Vis spectrophotometer (Hitachi).

\section{Fluorescence spectroscopy}

Fluorescence spectra were recorded on a Cary Eclipse Fluorescence Spectrophotometer. In a typical experiment, compounds were dissolved in methanol. The spectra were recorded by exciting the system at $270 \mathrm{~nm}$, and $274 \mathrm{~nm}$.

\section{Atomic force microscopy}

For AFM, images were taken with an NT-MDT instrument (model no. AP-0100), in the semi-contact mode.

\section{Field emission scanning electron microscopy}

Morphologies of the reported compounds were investigated using field emission-scanning electron microscopy (FE-SEM). A small amount of solution $(0.02 \mathrm{M})$ of the compound was placed on a clean glass slide and then dried by slow evaporation. The sample was then allowed to dry under vacuum at $30{ }^{\circ} \mathrm{C}$ for $24 \mathrm{~h}$. The materials were gold-coated, and the micrographs were taken in an FE-SEM apparatus (Zeiss DSM 950 scanning electron microscope).

\section{Conflicts of interest}

There are no conflicts to declare.

\section{Acknowledgements}

S. Kumar and S. K. Nandi acknowledge the CSIR, India, for a research fellowship. S. Bera thanks UGC, India, for a fellowship. We acknowledge the IISER Kolkata, India, for financial assistance.

\section{Notes and references}

1 D. Xia, P. Wang, X. Ji, N. M. Khashab, J. L. Sessler and F. Huang, Chem. Rev., 2020, 120, 6070-6123. 
2 G. Prieto, H. Tüysüz, N. Duyckaerts, J. Knossalla, G. H. Wang and F. Schüth, Chem. Rev., 2016, 116, 14056-14119.

3 D. Ni, L. Wang, Y. Sun, Z. Guan, S. Yang and K. Zhou, Angew. Chem., Int. Ed., 2010, 49, 4223-4227.

4 W. Kim, J. Thvenot, E. Ibarboure, S. Lecommandoux and E. L. Chaikof, Angew. Chem., Int. Ed., 2010, 49, 4257-4260.

5 H. Kim, T. Kim and M. Lee, Acc. Chem. Res., 2011, 44, 72-82.

6 A. S. Mahadevi and G. N. Sastry, Chem. Rev., 2016, 116, 2275-2825.

7 K. M. Dethlefs and P. Hobza, Chem. Rev., 2000, 100, 143-168.

8 (a) T. Xiao, L. Xu, J. Wang, Z. Y. Li, X. Q. Sun and L. Wang, Org. Chem. Front., 2019, 6, 936-941; (b) T. Xiao, L. Xu, J. Götz, M. Cheng, F. Würthner, J. Gu, X. Feng, Z. Y. Li, X. Q. Sun and L. Wang, Mater. Chem. Front., 2019, 3, 2738-2745.

9 X. Du, J. Zhou, J. Shi and B. Xu, Chem. Rev., 2015, 115, 13165-13307.

10 R. Klajn, J. F. Stoddart and B. A. Grzybowski, Chem. Soc. Rev., 2010, 39, 2203-2237.

11 A. Ajayaghosh and V. K. Praveen, Acc. Chem. Res., 2007, 40, 644-656.

12 C. de las Heras Alarcón, S. Pennadam and C. Alexander, Chem. Soc. Rev., 2005, 34, 276-285.

13 P. M. Mendes, Chem. Soc. Rev., 2008, 37, 2512-2529.

14 S. Yagai and A. Kitamura, Chem. Soc. Rev., 2008, 37, 1520-1529.

15 S.-I. Kawano, N. Fujita and S. Shinkai, J. Am. Chem. Soc., 2004, 126, 8592-8593.

16 (a) B. S. Pattni, V. V. Chupin and V. P. Torchilin, Chem. Rev., 2015, 115, 10938-10966; (b) T. Xiao, W. Zhong, L. Xu, X. Q. Sun, X. Y. Hu and L. Wang, Org. Biomol. Chem., 2019, 17, 1336-1350; (c) T. Xiao, L. Qi, W. Zhong, C. Lin, R. Wang and L. Wang, Mater. Chem. Front., 2019, 3, 1973-1993.

17 (a) P. Vitovič, J. M. Alakoskela and P. K. J. Kinnunen, J. Med. Chem., 2008, 51, 1842-1848; (b) P. George, J. Appl. Pharm. Sci., 2011, 1, 40-44; (c) W. G. H. Steve and E. Unger, Chem. Res. Toxicol., 2006, 19, 1564-1569; (d) T. Liu and R. B. Altman, J. Chem. Inf. Model., 2015, 55, 1483-1494.

18 (a) M. Karimi, A. Ghasemi, P. S. Zangabad, R. Rahighi, S. M. M. Basri, H. Mirshekari, M. Amiri, Z. S. Pishabad, A. Aslani, M. Bozorgomid, D. Ghosh, A. Beyzavi, A. Vaseghi, A. R. Aref, L. Haghani, S. Bahramia and M. R. Hamblin, Chem. Soc. Rev., 2016, 45, 1457-1501; (b) S. Kalepu and V. Nekkanti, Acta Pharm. Sin. B, 2015, 5, 442-453; (c) R. Xing, T. Jiao, L. Yan, G. Ma, L. Liu, L. Dai, J. Li, H. Möhwald and X. Yan, ACS Appl. Mater. Interfaces, 2015, 7, 24733-24740; (d) Q. Zou, L. Zhang, X. Yan, A. Wang, G. Ma, J. Li, H. Möhwald and S. Mann, Angew. Chem., Int. Ed., 2014, 53, 2366-2370.

19 (a) E. K. Lim, T. Kim, S. Paik, S. Haam, Y.-M. Huh and K. Lee, Chem. Rev., 2015, 115, 327-394; (b) M. Karimi, A. Ghasemi, P. S. Zangabad, R. Rahighi, S. M. M. Basri, H. Mirshekari, M. Amiri, Z. S. Pishabad, A. Aslani, M. Bozorgomid, D. Ghosh, A. Beyzavi, A. Vaseghi, A. R. Aref, L. Haghani, S. Bahrami and M. R. Hamblin, Chem. Soc. Rev., 2016, 45, 1457-1501; (c) H. Zhang, J. Fei, X. Yan, A. Wang and J. Li, Adv. Funct. Mater., 2015, 25, 1193-1204.
20 (a) A. O. Eniola and D. A. Hammer, J. Controlled Release, 2003, 87, 15-22; (b) S. S. Chandran, A. Nan, D. M. Rosen, H. Ghandehari and S. R. Denmeade, Mol. Cancer Ther., 2007, 6, 2928-2937; (c) F. Gu, L. Zhang, B. A. Teply, N. Mann, A. Wang, A. F. Radovic-Moreno, R. Langer and O. C. Farokhzad, Proc. Natl. Acad. Sci. U. S. A., 2008, 105, 2586-2591.

21 (a) S. Sengupta, A. Goswami and R. Mondal, New J. Chem., 2014, 38, 2470-2479; (b) J. Zhuang, C.-H. Kuo, L. Y. Chou, D. Y. Liu, E. Weerapana and C. K. Tsung, ACS Nano, 2014, 8, 2812-2819; (c) K. T. Holman, Angew. Chem., Int. Ed., 2011, 50, 1228-1230.

22 (a) J. Qi, X. Lai, J. Wang, H. Tang, H. Ren, Y. Yang, Q. Jin, L. Zhang, R. Yu, G. Ma, Z. Su, H. Zhao and D. Wang, Chem. Soc. Rev., 2015, 44, 6749-6773; (b) M. Dogru, A. Sonnauer, S. Zimdars, M. Döblinger, P. Knochel and T. Bein, CrystEngComm, 2013, 15, 1500-1502.

23 (a) Z. Liu, K. Chen, C. Davis, S. Sherlock, Q. Cao, X. Chen and H. Dai, Cancer Res., 2008, 68, 6652-6660; (b) W. Wu, S. Wieckowski, G. Pastorin, M. Benincasa, C. Klumpp, J. P. Briand, R. Gennaro, M. Prato and A. Bianco, Angew. Chem., Int. Ed., 2005, 44, 6358-6362; (c) S. Kumar, R. Rani, N. Dilbaghi, K. Tankeshwar and K.-H. Kim, Chem. Soc. Rev., 2017, 46, 158-196.

24 C. R. Patra, R. Bhattacharya, D. Mukhopadhyay and P. Mukherjee, Adv. Drug Delivery Rev., 2010, 62, 346-361.

25 M. R. Mohammadi, A. Nojoomi, M. Mozafari, A. Dubnika, M. Inayathullah and J. Rajadas, J. Mater. Chem. B, 2017, 5, 3995-4018.

26 (a) S. Laurent, D. Forge, M. Port, A. Roch, C. Robic, L. V. Elst and R. N. Muller, Chem. Rev., 2008, 108, 2064-2110; (b) A. K. Gupta and M. Gupta, Biomaterials, 2005, 26, 3995-4021.

27 (a) L. M. Kaminskas, B. D. Kelly, V. M. McLeod, G. Sberna, D. J. Owen, B. J. Boyd and C. J. Porter, J. Controlled Release, 2011, 152, 241-248; (b) S. C. Zimmerman, J. R. Quinn, E. Burakowska and R. Haag, Angew. Chem., Int. Ed., 2007, 46, 8164-8167; (c) H. T. Chen, M. F. Neerman, A. R. Parrish and E. E. Simanek, J. Am. Chem. Soc., 2004, 126, 10044-10048.

28 (a) B. S. Zolnik, A. G. Fernandez, N. Sadrieh and M. A. Dobrovolskaia, Endocrinology, 2010, 151, 458-465; (b) L. A. Mitchell, F. T. Lauer, S. W. Burchiel and J. D. McDonald, Nat. Nanotechnol., 2009, 4, 451-456.

29 (a) S. Cao, Z. Pei, Y. Xu and Y. Pei, Chem. Mater., 2016, 28, 4501-4506; (b) D. G. Fatouros, D. A. Lamprou, A. J. Urquhart, S. N. Yannopoulos, I. S. Vizirianakis, S. Zhang and S. Koutsopoulos, ACS Appl. Mater. Interfaces, 2014, 6, 8184-8189; (c) C. Cai, J. Lin, Y. Lu, Q. Zhang and L. Wang, Chem. Soc. Rev., 2016, 45, 5985-6012.

30 (a) A. J. V. Hell, M. M. Fretz, D. J. A. Crommelin, W. E. Hennink and E. Mastrobattista, J. Controlled Release, 2010, 141, 347-353; (b) C. Yan and D. J. Pochan, Chem. Soc. Rev., 2010, 39, 3528-3540; (c) M. Reches and E. Gazit, Science, 2003, 300, 625-627; (d) J. D. Hartgerink, E. Beniash and S. I. Stupp, Science, 2001, 294, 1684-1688; (e) A. J. V. Hell, C. I. C. A. Costa, F. M. Flesch, M. Sutter, 
W. Jiskoot, D. J. A. Crommelin, W. E. Hennink and E. Mastrobattista, Biomacromolecules, 2007, 8, 2753-2761.

31 C. H. Gorbitz, M. Nilsen, K. Szeto and L. W. Tangen, Chem. Commun., 2005, 4288-4290.

32 Y. Ma, D. Liu, D. Wang, Y. Wang, Q. Fu, J. K. Fallon, X. Yang, Z. He and F. Liu, Mol. Pharmaceutics, 2014, 11, 2623-2630.

33 C. Sung, J. E. Raeder and E. W. Merrill, J. Pharm. Sci., 1990, 79, 829-834.

34 A. Hughes, M. J. Rogers, A. I. Idris and J. C. Crockett, Calcif. Tissue Int., 2007, 81, 403-413.

35 Y. Chen, T. Li, J. Li, S. Cheng, J. Wang, C. Verma, Y. Zhao and C. Wu, Org. Biomol. Chem., 2017, 15, 1921-1929.

36 S. Dinda, M. Ghosh and P. K. Das, Langmuir, 2016, 32, 6701-6712.

37 (a) M. Debnath, S. Sasmal and D. Haldar, J. Mater. Chem. B, 2017, 5, 5450-5457; (b) S. K. Maity, S. Bera, A. Paikar,
A. Pramanik and D. Haldar, CrystEngComm, 2014, 16, 2527-2534; (c) S. K. Maity, S. Bera, A. Paikar, A. Pramanik and D. Haldar, CrystEngComm, 2013, 15, 5860-5866; (d) S. Maity, P. Jana, S. K. Maity and D. Haldar, Langmuir, 2011, 27, 3835-3841; (e) S. Maity, P. Jana, S. K. Maity and D. Haldar, Soft Matter, 2011, 7, 10174-10181; $(f)$ A. Pramanik, A. Paikar and D. Haldar, RSC Adv., 2015, 5, 53886-53892.

38 J. J. van Grop, J. A. J. M. Vekemans and E. W. Meijer, J. Am. Chem. Soc., 2002, 124, 14759-14769.

39 (a) C. Toniolo and M. Palumbo, Biopolymers, 1977, 16, 219-224; (b) V. Moretto, M. Crisma, G. M. Bonora, C. Toniolo, H. Balaram and P. Balaram, Macromolecules, 1989, 22, 2939-2944.

40 M. Sangra, S. Jaiswal and G. D. Gupta, Indo Am. J. Pharm. Res., 2014, 4, 2943-2950. 